\title{
Quasilinear theory of terahertz free-electron lasers based on Compton scattering of incoherent pump wave by intense relativistic electron beam
}

\author{
N. S. Ginzburg and E. R. Kocharovskaya \\ Institute of Applied Physics of the Russian Academy of Sciences, \\ 46 Ulyanov Street, 603950 Nizhny Novgorod, Russia \\ (Received 25 January 2016; published 2 August 2016)
}

\begin{abstract}
The use of incoherent broadband pump radiation for improving the electron efficiency in the freeelectron lasers (FEL) based on stimulated backscattering is considered. On the basis of a quasilinear approach, it is shown that the efficiency increases in proportion to the width of the pump spectrum. The effect is owing to a broadening of the spectrum of synchronous combination waves and realization of a mechanism of stochastic particle deceleration. The injection of a monochromatic seed signal in a single pass FEL amplifier or the implementation of a selective high-Q resonator in an FEL oscillator makes the high-frequency scattered radiation be monochromatic in spite of an incoherent pumping. In the regime of stochastic particle deceleration, the efficiency only slightly depends on the spread of the beam parameters, which is beneficial for a terahertz FEL powered by intense relativistic electron beams.
\end{abstract}

DOI: 10.1103/PhysRevAccelBeams.19.080701

\section{INTRODUCTION}

Terahertz waves are promising for diagnostics and spectroscopy of various media, including the development of high-resolution electron paramagnetic resonance (EPR) and nuclear magnetic resonance (NMR) spectroscopy. Powerful terahertz radiation can be used to create a dense plasma and control its parameters. Recently a lot of attention has been paid to the development of a different type of sources operating at terahertz band at a high power level. Alongside with promotion of some traditional devices like gyrotons [1,2], Cherenkov devices [3-5] and FELs based on the stimulated undulator emission [6-8] to this wave band, some new schemes are examined. Among such schemes, we mention the generation of $\mathrm{THz}$ pulses during the nonlinear interaction of femtosecond laser pulses with a different medium including semiconductors and plasmas [9-14].

In this paper, we consider one more possible scheme of generation and amplification of powerful $\mathrm{THz}$ radiation based on Compton backscattering of a strong lowfrequency pumping wave on high-current relativistic electron beams. Formed by explosive emission cathodes, such beams have considerable powers and current densities, but at the same time are characterized by a large spread of parameters, which generally precludes their use for the efficient generation of short-wavelength radiation. In order to exploit the potential of intense beams more effectively, we suggest using incoherent pump radiation [15-18]. The

Published by the American Physical Society under the terms of the Creative Commons Attribution 3.0 License. Further distribution of this work must maintain attribution to the author(s) and the published article's title, journal citation, and DOI. advantage of the incoherent pumping compared to the monochromatic one traditionally considered for such systems (which are called Compton free-electron lasers [19-24]) is the expansion of the spectrum of synchronous combination waves and the realization of a mechanism of diffusive stochastic deceleration of the electrons. As a result, the efficiency of conversion of the beam kinetic energy into the energy of short-wavelength scattered radiation is proportional to the width of the pump spectrum. Moreover, this efficiency only slightly depends on the width of an electron distribution function. It should be noted that in spite of incoherent pumping, the injection of a monochromatic seed signal in an amplifier configuration [Fig. 1(a)] or the implementation of a selective high-Q resonator in an oscillator [Fig. 1(b)] can guarantee almost

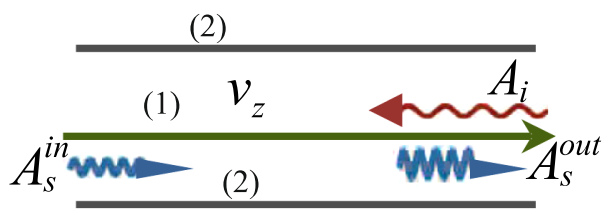

(a)

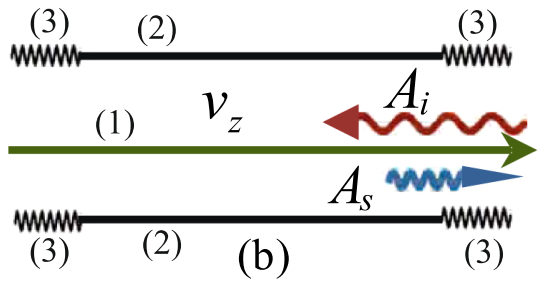

FIG. 1. Schematic of amplifier (a) and generator (b) with incoherent pump wave. (1) Electron beam; (2) waveguide; (3) Bragg reflectors. 
monochromatic spectrum of the high-frequency scattered radiation. A description of the considered type of Compton FEL may be based on a quasilinear theory [25-27] because the electrons interact with a broad spectrum of the combination waves, which is proportional to the width of the pump wave spectrum. It should be noted that previously such a quasilinear approach has been employed for a theoretical analysis of FEL amplifiers [28-31] and FEL oscillators [32-34] with a monochromatic pump wave or a single periodical undulator field but a multifrequency signal wave.

In Sec. II we derive basic quasilinear equations which describe the Compton FEL with incoherent pump radiation. In Sec. III this approach is applied to the analysis of a high gain single-pass amplifier with a seed signal. In Sec. IV we generalize the model and analyze a Compton FEL oscillator with a high-Q resonator.

\section{BASIC EQUATIONS}

Let us assume that a monochromatic signal wave propagates together with an electron beam and is described with a vector potential

$$
A_{s}=\operatorname{Re}\left\{x_{0} A_{s}(z) \exp \left[i\left(\omega_{s} t-\kappa_{s} z\right)\right]\right\} .
$$

In the amplification scheme, the frequency of this wave is determined by an external source. Let us consider a polychromatic pump field, so that its spectrum consists of a set of discrete lines with random phases

$$
A_{i}=\operatorname{Re}\left\{\sum_{m=0}^{\infty} x_{0} A_{i m}(z) \exp \left[i\left(\omega_{i m} t+\kappa_{i m} z\right)\right]\right\} .
$$

We assume that a spectral distance between neighboring harmonics $\delta \kappa_{i}$ is much smaller than the total width of the packet $\Delta \kappa_{i}$ and the amplitudes $A_{i m}$ are slowly varying functions with respect to a longitudinal coordinate $z$.

Under the combination synchronism conditions

$$
\omega_{s}-\kappa_{s} v_{z} \approx \omega_{i m}+\kappa_{i m} v_{z},
$$

the averaged motion of the relativistic electrons in the signal and pump fields can be described using the kinetic equation for a one-dimensional distribution function [19]:

$$
\frac{\partial f}{\partial t}+v_{z} \frac{\partial f}{\partial z}+F_{z} \frac{\partial f}{\partial p_{z}}=0 .
$$

Here

$F_{z}=\frac{e^{2}}{2 \mathrm{E}} \operatorname{Re}\left\{\sum_{m=0}^{\infty} i \kappa_{c m} A_{s} A_{i m}^{*}(z) \exp \left[i\left(\omega_{c m} t-\kappa_{c m} z\right)\right]\right\}$

is the averaged ponderomotive force, $\kappa_{c m}=\kappa_{s}+\kappa_{i m}$ and $\omega_{c m}=\omega_{s}-\omega_{i m}$ are the wave numbers and frequencies of the ensemble of combination waves, $v_{z}, p_{z}=m v_{z} \gamma$ and $\mathrm{E}=m c^{2} \gamma$ are the longitudinal velocity, momentum, and energy of the electrons $\gamma=\left(1-v^{2} / c^{2}\right)^{-1 / 2}$ is the Lorenz factor.

After traditional for the quasilinear approximation [25-27] averaging of Eq. (2) over oscillations caused by the action of separate components from the ensemble of combination waves, the equation for a slowly varying distribution function $\bar{f}(z, p)$ takes the form

$v_{z} \frac{\partial \bar{f}}{\partial z}=\frac{\partial}{\partial p_{z}}\left(D\left(z, p_{z}\right) \frac{\partial \bar{f}}{\partial p_{z}}\right),\left.\quad \bar{f}\right|_{z=0}=\bar{f}^{0}\left(p_{z}\right)$,

with the diffusion coefficient

$$
D=\left.\frac{8 \pi^{3} e^{4} c^{2} \kappa_{c}^{2} I_{s} I_{i}\left(\kappa_{i}\right)}{\omega_{s}^{2} \omega_{i}^{2}\left(v_{z}+c\right) \mathrm{E}^{2}}\right|_{\kappa_{i}=\kappa_{s}\left(c-v_{z}\right) /\left(c+v_{z}\right)} .
$$

Here $\bar{f}^{0}\left(p_{z}\right)$ is the initial longitudinal momentum distribution function of the electrons, $I_{s}=\left|A_{s}\right|^{2} \omega_{s}^{2} / 8 \pi c$ is the intensity of the signal wave. Assuming that the phases of spectral components in pump radiation are random, this field can be described by the spectral density $I_{i}=\left|A_{i}\right|^{2} \omega_{i}^{2} / 8 \pi c \delta \kappa$. Spatial dependence of intensities of the signal and pumping waves obeys the equations

$\frac{d I_{s}}{d z}=\left.\frac{\pi^{2} e^{2} \omega_{p}^{2}}{m c^{5}} \frac{I_{s}}{\kappa_{s}} \int_{0}^{\infty} \frac{I_{i}}{\omega_{i}^{2}}\left(\mathrm{E} \frac{\partial \bar{f}}{\partial p_{z}}\right)\right|_{v_{z}=v_{c}} d \kappa_{i},\left.\quad I_{s}\right|_{z=0}=I_{s}^{0}$,

$\frac{d I_{i}}{d z}=\left.\frac{\pi^{2} e^{2} \omega_{p}^{2}}{m c^{5}} \frac{I_{i}}{\kappa_{i}} \frac{I_{s}}{\omega_{s}^{2}}\left(\mathrm{E} \frac{\partial \bar{f}}{\partial p_{z}}\right)\right|_{v_{z}=v_{c}},\left.\quad I_{i}\right|_{z=l}=I_{i}^{0}$,

where $v_{c}=\omega_{c} / k_{c}$ is the phase velocity of the combination waves, $\omega_{p}=\left(4 \pi e^{2} N_{0} / m\right)^{1 / 2}$ is the electron plasma frequency, $N_{0}$ is the electron beam density, and $l$ is the length of the scattering region.

The system of equations (4)-(6) has the integrals

$$
\begin{gathered}
K-I_{s}+\int_{0}^{\infty} I_{i} d \kappa_{i}=\mathrm{const}, \\
\int_{0}^{\infty} \frac{I_{i} d \kappa_{i}}{\kappa_{i}}-\frac{I_{s}}{\kappa_{s}}=\mathrm{const}
\end{gathered}
$$

which are the conservation laws of the energy and the number of quanta in the scattering process, respectively. Here $K=N_{0} \int_{0}^{\infty} v_{z} \mathrm{E} \bar{f} d p_{z}$ is the kinetic energy flux of the electrons. As follows from Eqs. (7) and (8), in the case of large Doppler frequency up-conversion $\left(\hbar \omega_{s} \gg \hbar \omega_{i}\right)$, the radiation energy is drawn mainly from the electron beam $\left(\Delta I_{s} \simeq \Delta K\right)$, and the depletion of the pump radiation (when its intensity is sufficiently large) may be neglected. 


\section{HIGH GAIN SINGLE-PASS AMPLIFIER}

Under the assumption of a fixed pump field amplitude for the ultrarelativistic limit $\left(\gamma \gg 1, p_{z} \simeq m c \gamma\right)$ the system of Eqs. (4)-(6) has the form that describes single-pass amplification of the signal wave:

$$
\begin{gathered}
\frac{\partial F}{\partial z}=\frac{\partial}{\partial \gamma}\left(\tilde{D} \frac{\partial F}{\partial \gamma}\right),\left.\quad F\right|_{z=0}=F_{0}(\gamma), \\
\frac{\partial P_{s}}{\partial z}=\frac{\pi \omega_{p}^{2}}{2 \omega_{s}^{2}} \int_{1}^{\infty} \tilde{D} \frac{\partial F}{\partial \gamma} d \gamma,\left.\quad P_{s}\right|_{z=0}=P_{s}^{0},
\end{gathered}
$$

where $\quad \tilde{D}=4 P_{s} P_{i} \kappa_{s}^{2} \gamma^{2} / \pi, \quad P_{s}=I_{s} \lambda_{s}^{2} / P_{*} \quad$ is the power of the signal wave in absolute power units $\left(P_{*}=m^{2} c^{5} / e^{2}=8.7 \mathrm{GW}\right)$ passing through an area equal to $\lambda_{s}^{2}$, and $P_{i}=I_{i} \lambda_{s}^{2} /\left(P_{*} \delta \kappa_{i}\right)$ is the spectral power of the pump radiation in the same normalization. $F(\gamma, z)=\bar{f} m c$ is the electron energy distribution function. It should be noted that we replaced the integration over pump wave numbers $\kappa_{i}$ in Eq. (5) with the integration over $\gamma$ in Eq. (10) using the relation: $\kappa_{i}=\kappa_{s} / 4 \gamma^{2}$ that follows from the synchronism condition (1) for the ultrarelativistic electron beam.

\section{A. Small signal regime}

In the linear approximation, we can neglect perturbation of the energy distribution function and reduce Eq. (10) to the form

$$
\frac{\partial P_{s}}{\partial Z}=\Lambda P_{s},
$$

where $Z=4 \pi \omega_{p}^{2} \bar{\gamma}^{2} P_{i}^{\text {tot }} \lambda_{i} z / \omega_{s}^{2}$ is the normalized longitudinal coordinate,

$$
\Lambda=\int_{0}^{\infty} u^{2} g(u) \frac{\partial \hat{F}_{0}(u)}{\partial u} d u
$$

is the normalized spatial growth rate, and $u=\gamma / \bar{\gamma}, \bar{\gamma}$ is the mean electron energy. In (12) we presented the spectral distribution of the pump radiation as $P_{i}(\gamma)=P_{i}^{\text {tot }} g(\gamma) / \bar{\kappa}_{i}$, where $P_{i}^{\text {tot }}$ is the total dimensionless power, $\bar{\kappa}_{i}$ is the central harmonic of the pump wave packet, and $g(\gamma)$ is the function which describes spectral profile. Obviously, the growth rate is determined by the unperturbed electron distribution function $\widehat{F}_{0}(u)$ and a pump spectrum profile $g(u)$.

Further, we will describe the unperturbed electron distribution and the pump spectrum profile by the functions [see Fig. 2(a)]

$$
\begin{aligned}
\widehat{F}_{0}(u) & =\frac{1}{\sqrt{2 \pi} \delta} \exp \left(-\frac{(1-u)^{2}}{2 \delta^{2}}\right), \\
g(u) & =\frac{1}{\sqrt{2 \pi} \sigma} \exp \left[-\left(\frac{(1-\varepsilon)^{2}-u^{2}}{\sqrt{2} u^{2} \sigma}\right)^{2}\right] .
\end{aligned}
$$

Here $\delta$ is the electron energy dispersion (relative energy spread $\Delta \gamma / \bar{\gamma}=2 \delta), \sigma$ is the half-width of the pump

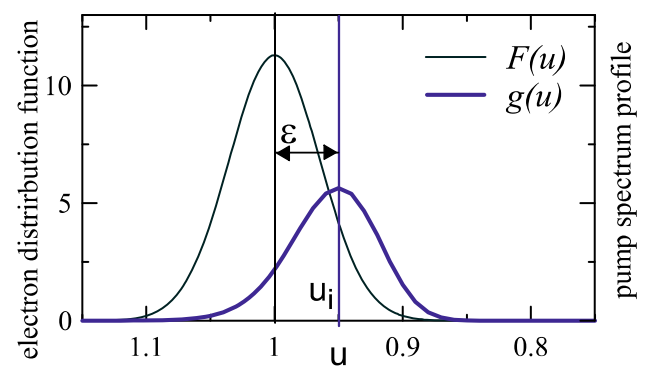

(a)

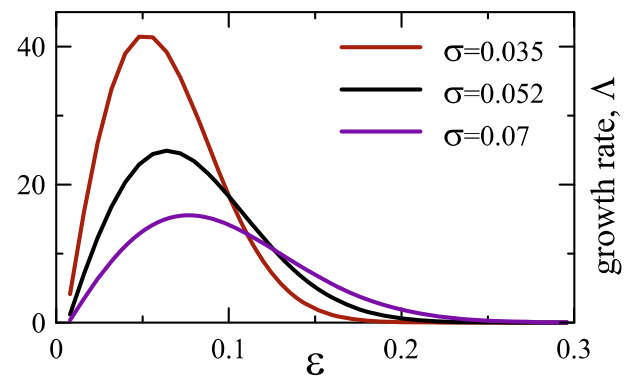

(b)

FIG. 2. Single-pass amplifier. (a) Mutual arrangement of electron distribution function $F(u)$ with initial energy dispersion $\delta=0.035$ and profile of pump spectrum (blue line) with a halfwidth $\sigma=0.07$ in the energy space. The shift between the center of the pump spectrum and the center of the electron distribution function is $\varepsilon=1-u_{i}=0.05$. (b) Dependence of spatial growth rate $\Lambda$ on relative shift $\varepsilon$ for different values of the pump spectrum half-width $\sigma$ calculated for electron energy dispersion $\delta=0.035$.

spectrum, and the parameter $\varepsilon=1-u_{i}$ characterizes the relative shift between the centers of the electron distribution function and the pump wave spectrum where $u_{i}=$ $\sqrt{\kappa_{s} / 4 \bar{\gamma}^{2} \bar{\kappa}_{i}}$ corresponds to the central line $\bar{\kappa}_{i}$ of this spectrum. It should be noted that function $g(u)$ just corresponds to the approximation of the pump wave spectrum $P_{i}\left(k_{i}\right)$ by a Gaussian function.

In the case of a coherent monochromatic pump wave: $\sigma \rightarrow 0$, when $g(u)$ tends to a delta-function $\delta\left(u_{i}-u\right)$; from (12) we get a well-known expression for the growth rate which describes the kinetic stage of the FEL instability

$$
\Lambda=\left.u_{i}^{2} \frac{\partial \widehat{F}_{0}}{\partial u}\right|_{u=u_{i}}=\frac{\varepsilon(1-\varepsilon)^{2}}{\delta^{3} \sqrt{\pi}} \exp \left(-\frac{\varepsilon^{2}}{2 \delta^{2}}\right) .
$$

Equation (14) demonstrates the drop of the growth rate with increasing electron energy dispersion $\delta$.

In the case of the incoherent pump radiation, the dependence of the growth rate $\Lambda(\varepsilon)$ for different widths of the pump spectrum is shown in Fig. 2(b). Obviously, the growth rate decreases with the increase of the spectral width $\sigma$ and the energy dispersion $\delta$. Nevertheless, for fixed $\sigma$ and $\delta$ there is an optimal relative shift $\varepsilon$ between the pump spectrum and the electron energy distribution function. 


\section{B. Nonlinear stage}

To carry out the numerical simulation of the saturation process, it is convenient to take into account the energy conservation law (7) and transform Eqs. (9) and (10) to a single diffusion equation containing a minimal number of independent parameters:

$$
\frac{\partial \widehat{F}}{\partial Z}=\frac{\partial}{\partial u}\left(\left(\bar{P}_{s}^{0}+\eta\right) g(u) u^{2} \frac{\partial \widehat{F}}{\partial u}\right) .
$$

Here, $\bar{P}_{s}^{0}=2 \omega_{s}^{2} P_{s}^{0} / \pi \omega_{p}^{2} \bar{\gamma}^{2}$ is the normalized intensity of the initial signal, and

$$
\eta=1-\int_{0}^{\infty} u \widehat{F} d u
$$

is the electron efficiency that defines the rate of the energy conversion from the relativistic electron beam to the signal wave.

The results of the numerical solution of Eq. (15) presented in Fig. 3 show that for fixed total power of the pump the electron efficiency increases with the growth of its spectrum width. Thus, when the relative half-width of the pump spectrum is $\sigma=0.07$, the maximum efficiency reaches $5 \%$. At the same time, an increase in the width of the pump spectrum leads to a drop in the spatial growth rate $\Lambda$, and, accordingly, the length of the interaction space, for which the maximum efficiency is achieved, increases [Fig. 3(b)]. The results of the numerical simulations confirm the assumption that the method for efficiency increasing based on using the incoherent pump is not critical in regard to the quality of the electron beam. As seen from Fig. 4 the value of the electron energy dispersion $\delta$ is only slightly affected by the maximum efficiency. Moreover, in the considered stochastic deceleration regime the maximal efficiency also very slightly depends on the integrated intensity of the pump radiation and the electron beam density. In fact, final gain saturation is caused by the formation of a plateau on the electron distribution function [Fig. 3(a)], whose width and, therefore, maximum efficiency are determined basically by the width of the pump spectrum $\sigma$ as well as by the distance $\varepsilon$ (in the energy space) between the center of the pump wave line and the center of the particle distribution function. The pump intensity and the electron beam density influence mainly the growth rate and the length of the scattering region. It is clear that in order to increase efficiency the pump spectrum should be shifted [Fig. 2(a)] to the region that corresponds to the energies of resonance electrons smaller than the energy of an electron $\bar{\gamma}$ which belongs to the center of the distribution function $\widehat{F}(u)$.

Let us evaluate (see Table I) the possibilities of using the considered mechanism for effective amplification of submillimeter radiation by an intense relativistic beam with a current of $J=5 \mathrm{kA}$, a mean particle energy of $1.5 \mathrm{MeV}$

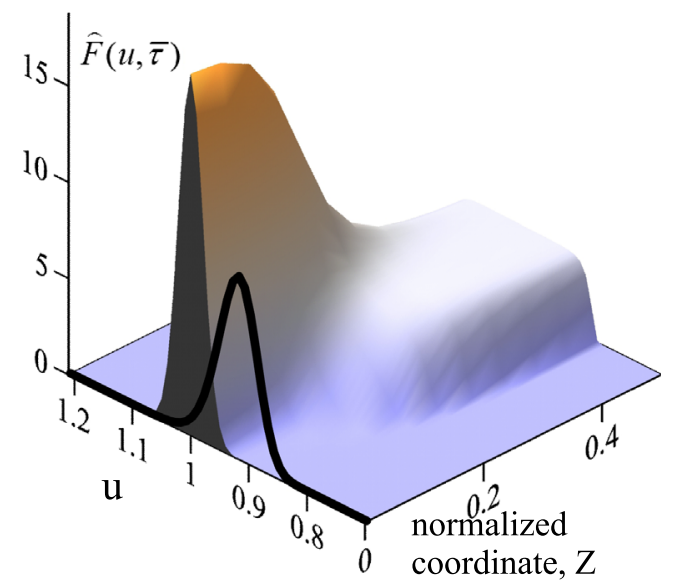

(a)

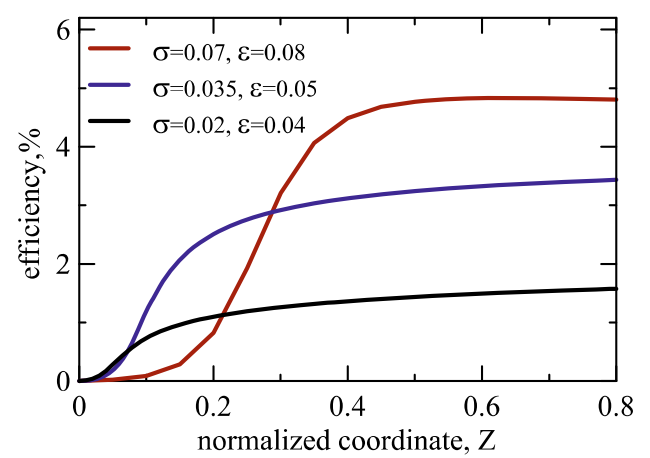

(b)

FIG. 3. (a) Spatial evolution of the electron distribution function $\widehat{F}(u, Z)$ for the initial electron energy dispersion $\delta=0.02$, pump spectrum half-width $\sigma=0.07$, and relative shift $\varepsilon=0.08$, which is optimal for efficiency. Pump spectrum profile is shown by the black line. (b) Dependences of the electron efficiency $\eta$ on the amplification length $Z$ for the different pump spectrum half-width $\sigma$ and the optimal relative shift $\varepsilon$.

$(\bar{\gamma}=4)$, and an energy spread $\Delta \gamma / \bar{\gamma}=4 \%(\delta=0.02)$. Let the mean wavelength of the pump field be $\lambda_{i}=3 \mathrm{~cm}$, the half-width of the spectrum be $\sigma=0.07$, and the total power be $1 \mathrm{GW}$. A vircator [35] or a relativistic backward-wave oscillator operating in the self-modulation stochastic regime [36,37] can, in principle, serve as the source of incoherent pump radiation. Gyrotrons with radiation power of $10 \mathrm{~kW}$ [2] can be used as the source of the input signal for the scattered radiation with a wavelength of $\lambda_{s}=0.45 \mathrm{~mm}$.

Under the assumption that the scattering process takes place in the waveguide with a cross-section of $2.5 \mathrm{~cm}^{2}$ the dependence of the electron efficiency on the longitudinal coordinate is shown in Fig. 3(b). At the linear stage for $\varepsilon=0.08$ the growth rate equals to $0.25 \mathrm{~cm}^{-1}$. The maximum efficiency of $\sim 5 \%$ corresponds to the output power of $\sim 375 \mathrm{MW}$ and is achieved at the length of the scattering region of $\sim 70 \mathrm{~cm}$. 


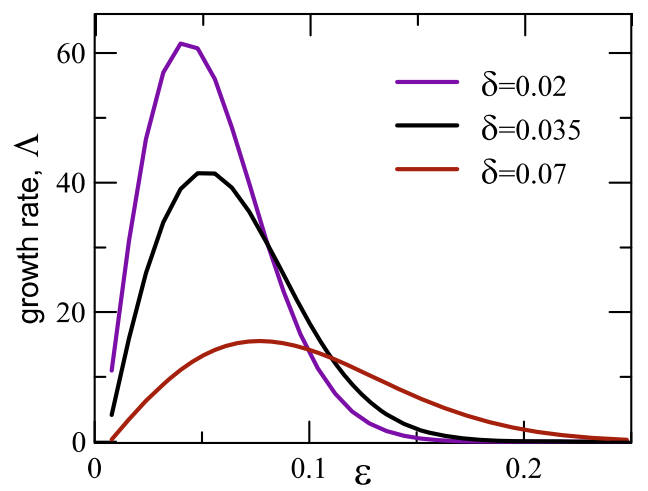

(a)

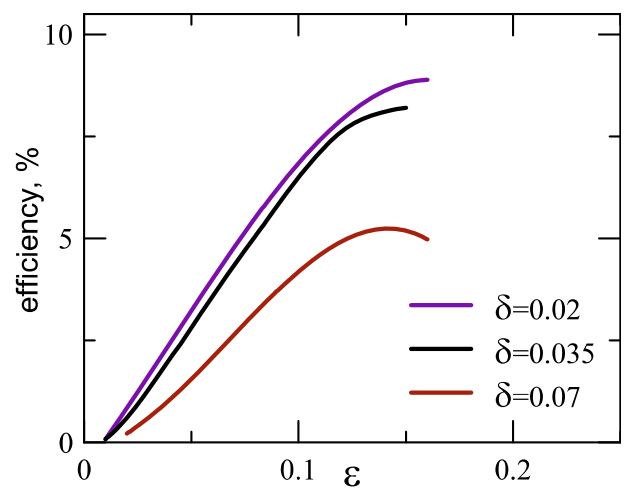

(b)

FIG. 4. Dependence of (a) spatial growth rate $\Lambda$ and (b) the electron efficiency $\eta$ on the shift $\varepsilon$ between the center of the pump wave spectrum and the center of the electron distribution function for a fixed half-width of pump spectrum $\sigma=0.07$ but different values of electron energy dispersion $\delta$.

\section{OSCILLATOR WITH HIGH-Q RESONATOR}

Let us consider further an oscillator scheme assuming that the scattered radiation is confined by two mirrors

TABLE I. Main parameters of the high-gain single-pass amplifier.

\begin{tabular}{lc}
\hline \hline Parameter & Value \\
\hline Beam current [kA] & 5 \\
Mean particle energy [MeV] & 1.5 \\
Energy spread, \% & 4 \\
Plasma frequency [c $\left.{ }^{-1}\right]$ & $5.7 \times 10^{10}$ \\
Mean wavelength of pump wave [cm] & 3 \\
Width of the spectrum, \% & 10 \\
Total power of pump [GW] & 1 \\
Signal wavelength [mm] & 0.45 \\
Power of input signal [kW] & 10 \\
Waveguide cross section [cm $\left.{ }^{2}\right]$ & 2.5 \\
Scattering region length [cm] & 70 \\
Growth rate [cm ${ }^{-1}$ ] & 0.25 \\
Output power [MW] & 375 \\
Electron efficiency, \% & 5 \\
\hline \hline
\end{tabular}

forming a high-Q resonator. At the $n$th run of the signal wave across the resonator, its amplification is described by Eq. (10). By integrating this equation over the longitudinal coordinate $z$ under the assumption that the distance between the mirrors $l$ coincides with the scattering region, we get for forward wave propagation

$$
P_{s+}^{n}(l)-P_{s+}^{n}(0)=\frac{\pi \omega_{p}^{2}}{2 \omega_{s}^{2}} \int_{0}^{l} \int_{1}^{\infty} \tilde{D} \frac{\partial F}{\partial \gamma} d \gamma d z .
$$

Due to the absence of interaction for backward wave propagation we have

$$
P_{s-}^{n}(l)=P_{s-}^{n}(0) .
$$

Then, taking into account reflections from the mirrors:

$$
P_{s+}^{n+1}(0)=R_{1} P_{s-}^{n}(0), \quad P_{s-}^{n}(l)=R_{2} P_{s+}^{n}(l),
$$

where $R_{1,2}$ are the reflection coefficients, we obtain the equation which defines the wave evolution

$$
\begin{aligned}
P_{s+}^{n+1}(l)-P_{s+}^{n}(l)= & -\left(1-R_{1} R_{2}\right) P_{s+}^{n}(l) \\
& +\frac{\pi \omega_{p}^{2}}{2 \omega_{s}^{2}} R_{1} R_{2} \int_{0}^{l} \int_{1}^{\infty} \tilde{D} \frac{\partial F}{\partial \gamma} d \gamma d z .
\end{aligned}
$$

In the case of the high-quality resonator $\left|1-R_{1} R_{2}\right| \ll 1$ and relatively low single pass gain, the variation of the field amplitude during the successive runs is small $\left|\left(P_{s+}^{n+1}-P_{s+}^{n}\right) / P_{s+}^{n}\right| \ll 1$. Under such conditions, we can substitute the finite-difference equation (17) with the differential equation

$$
\frac{d P_{s}}{d \tau}+\frac{\omega_{0}}{2 Q} P_{s}=\frac{\pi \omega_{p}^{2}}{2 \omega_{s}^{2}} \frac{v_{g r}}{2 l} \int_{0}^{l} \int_{1}^{\infty} \tilde{D} \frac{\partial F}{\partial \gamma} d \gamma d z
$$

where $Q=\omega_{0} l / v_{g r}\left(1-R_{1} R_{2}\right)$ is the quality factor, and $\tau=n \frac{2 l}{v_{g r}}$ is a slow time.

Equation (18) in combination with the diffusion equation (9) forms a self-consistent system describing FEL oscillators with the incoherent pump wave

$$
\begin{gathered}
\frac{d \tilde{P}_{s}}{d \bar{\tau}}+\tilde{P}_{s}=\int_{0}^{\tilde{L}} \int_{0}^{\infty} \tilde{P}_{s}(\bar{\tau}) g(u) u^{2} \frac{\partial \widehat{F}(u, \tilde{Z})}{\partial u} d u d \tilde{Z}, \\
\frac{\partial F}{\partial \tilde{Z}}=\frac{\partial}{\partial u}\left(\tilde{P}_{s}(\bar{\tau}) g(u) u^{2} \frac{\partial \widehat{F}}{\partial u}\right) .
\end{gathered}
$$

Here we introduce the following normalized variables: $\bar{\tau}=\tau \omega_{0} / 2 Q, \tilde{Z}=Z /\left(1-R_{1} R_{2}\right)$ is the longitudinal coordinate, $\tilde{L}=L /\left(1-R_{1} R_{2}\right)$ is the distance between the mirrors in the same normalization, $\tilde{P}_{s}=2 \omega_{s}^{2}\left(1-R_{1} R_{2}\right) P_{s} /$ $\pi \omega_{p}^{2} \bar{\gamma}^{2}$ is the power of the signal wave. 
For the high-Q resonator the time of the field variation strongly exceeds the electron transit time:

$$
T_{R}=\frac{Q}{\omega_{0}} \gg \frac{l}{v_{z}} .
$$

Under such conditions, we can integrate Eq. (20) for the distribution function over the longitudinal coordinate $\tilde{Z}$ by neglecting the dependence of the diffusion coefficient on this coordinate. Then, by integrating the excitation factor over the coordinate $\tilde{Z}$, we can find the value of the signal wave amplitude at the next time step from Eq. (19), and so on.

\section{A. Small signal regime}

Neglecting perturbation of the electron distribution function and looking for the solution of Eq. (19) in the form $\tilde{P}_{s} \sim \exp (\Gamma \tau)$, we get temporal growth rate in generation regime:

$$
\Gamma=\Lambda \tilde{L}-1
$$

For self-excitation condition from (21) we find

$$
\tilde{L}_{s t}=1 / \Lambda \text {. }
$$

In physical variables a threshold current density is given by the relation

$$
j_{s t}=\frac{J_{a}\left(1-R_{1} R_{2}\right)}{\Lambda P_{i}^{\mathrm{tot}} l \lambda_{s} \lambda_{i}^{2}},
$$

where $J_{a}=m c^{3} / e \simeq 17 \mathrm{kA}$ is the Alfven current. Obviously, the minimal resonator length $\tilde{L}_{s t}$ or minimal starting current (23) corresponds to the maximum of spatial growth rate.

\section{B. Nonlinear stage}

In the generation regime, deformation of the electron distribution function depends on the injection time and increases with the growth of the signal wave amplitude inside the resonator. Figure 5 demonstrates the process of steady-state oscillations onset at two different values of an excess of the electron current over the threshold. In the steady-state regime, the indicated deformation becomes more significant with the increase of the electron current [compare Figs. 5(a) and 5(b)]. In the case of a large excess, the formation of the plateau on the electron distribution function is evident. It should be noted that, due to the signal-wave energy accumulation in the resonator, the interaction length corresponding to a given level of the efficiency in the oscillator can be significantly shorter than the interaction length in the single-pass amplifier considered in Sec. III. Nevertheless, as in the amplifier, the efficiency can be increased by means of proper optimization of relative shift $\varepsilon$ between the center of the pump wave

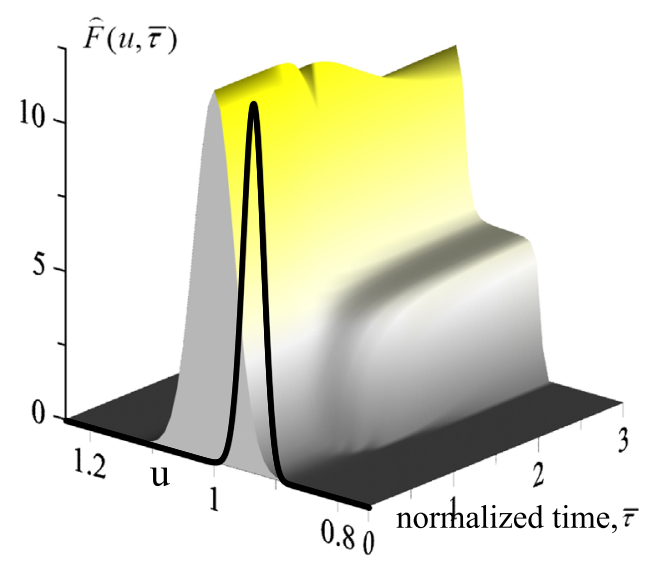

(a)

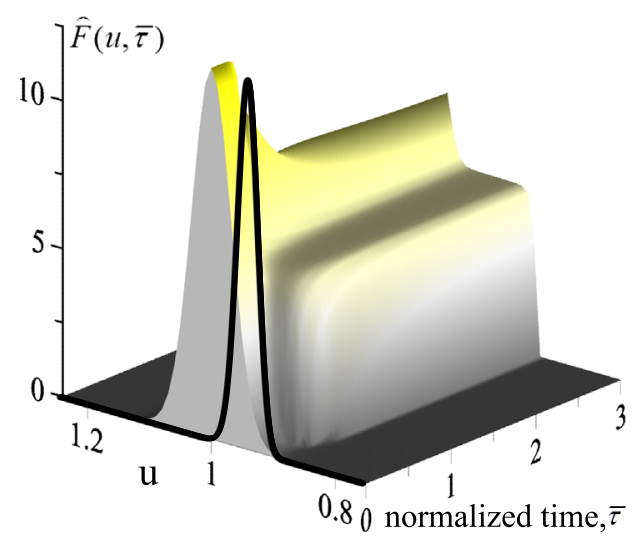

(b)

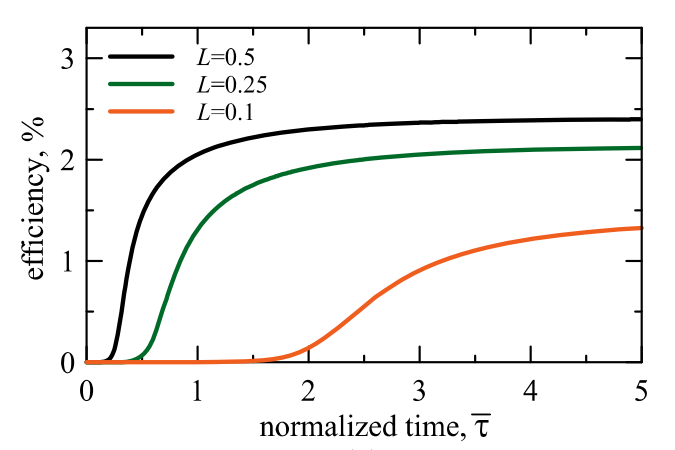

(c)

FIG. 5. Oscillator with the high-Q resonator. Temporal evolution of the electron distribution function $\bar{F}(u, \bar{\tau})$ for different resonator lengths (a) $\tilde{L}=0.25$, (b) $\tilde{L}=0.5$. Profile of pump spectrum is shown by the black line. (c) Corresponding dependences of electron efficiency on time $\bar{\tau}$ : $\delta=0.035, \sigma=0.035$, $\varepsilon=0.05$.

spectrum and the center of the electron distribution function (see Fig. 6).

Let us estimate (see Table II) the possibility of using the considered mechanism of backscattering for the generation of terahertz radiation by an intense relativistic beam with a current $J=450 A$, a mean particle energy $0.75 \mathrm{MeV}$ $(\bar{\gamma}=2.5)$, and an energy spread $\Delta \gamma / \bar{\gamma}=7 \%(\delta=0.035)$. 


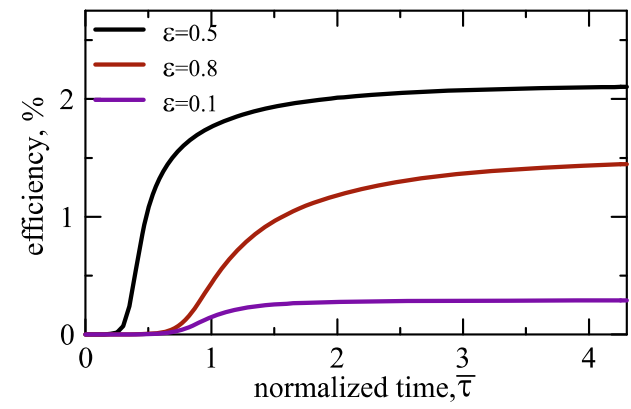

(a)

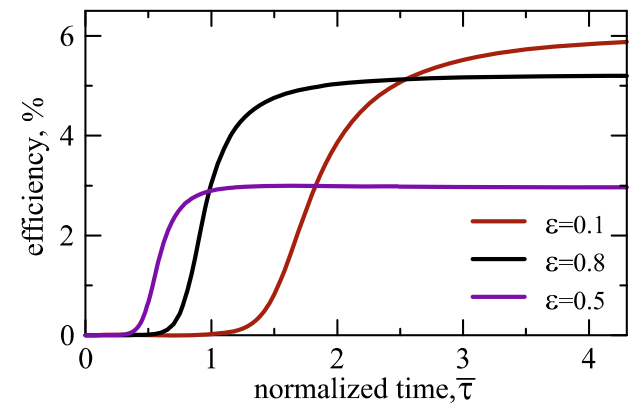

(b)

FIG. 6. Oscillator with the high-Q resonator. Temporal dependences of electron efficiency for the given values of the electron energy dispersion $\delta$ and the pump spectrum half-width $\sigma$ at the different values of relative shift $\varepsilon ; \tilde{L}=0.5$. (a) $\delta=0.035$, $\sigma=0.035$; (b) $\delta=0.02, \sigma=0.07$.

In the considered case we can take a $70 \mathrm{GHz}$ free-electron maser that is being developed at the accelerator ELMI (Budker Institute, Novosibirsk) [38,39] as a pump wave source. We assume that the total power of the pump wave is $100 \mathrm{MW}$, the spectrum width is $7 \%$ and the cross section of the interaction space is $4 \mathrm{~mm}^{2}$. For the chosen parameters the scattered radiation has a frequency of about $1.8 \mathrm{THz}$. As shown in $[40,41]$ two modified Bragg reflectors based on the coupling of cutoff and propagating modes can be used to compile a selective resonator for a signal wave of the

TABLE II. Main parameters of the oscillator.

\begin{tabular}{lc}
\hline \hline Parameter & Value \\
\hline Beam current [A] & 450 \\
Mean particle energy [MeV] & 0.75 \\
Energy spread, \% & 7 \\
Plasma frequency [c ${ }^{-1}$ ] & $8.1 \times 10^{10}$ \\
Mean frequency of pump wave [GHz] & 70 \\
Total power of pump [GW] & 0.1 \\
Waveguide cross section [mm ${ }^{2}$ ] & 4 \\
Scattering region length [cm] & 30 \\
Mirrors reflection coefficient & 0.9 \\
Generation frequency [THz] & 1.8 \\
Output power [MW] & 10 \\
Electron efficiency, $\%$ & 2 \\
\hline \hline
\end{tabular}

terahertz frequency range. Obviously, such a Bragg resonator is totally transparent for pump radiation.

For reflection coefficients $R=0.9$ according to formula (22), the normalized starting length is $L_{s t}=0.025$ that corresponds to the physical length of $3 \mathrm{~cm}$. For the length of the scattering region of $30 \mathrm{~cm}(\tilde{L}=0.25)$, as seen from Fig. 5(c), the electron efficiency amounts to $2 \%$ that corresponds to the power of terahertz radiation $\sim 10 \mathrm{MW}$.

\section{CONCLUSION}

The quasilinear theory of FEL based on Compton backscattering of incoherent pump radiation by the intense relativistic electron beam is developed. An injection of a monochromatic seed signal in a single pass FEL-amplifier or an implementation of a selective high-Q resonator in an FEL oscillator makes a high-frequency scattered radiation be monochromatic. It is demonstrated that the electron efficiency increases in proportion to the width of the pump spectrum which defines the spectrum of synchronous combination waves responsible for the stochastic particle deceleration. The efficiency in such a regime only slightly depends on the spread of the beam parameters.

We should note that up to the effects associated with depletion of the pump radiation, the physical processes in FELs based on stimulated wave scattering and stimulated undulator emission are identical since in the comoving reference frame each electron perceives a periodic undulator magnetic field as an electromagnetic pump wave. Developing this analogy, we can conclude that a random quasiperiodic magnetic field can be employed for the arrangement of stochastic particle deceleration and efficiency enhancement in FEL driven by electron beams with a large energy spread. It is also interesting to study the stochastic particle deceleration in application to $\mathrm{x}$-ray Compton FELs with optical pumping [19-21,24]. Here incoherent pumping can be realized by summation of radiation from a large number of powerful lasers [42].

\section{ACKNOWLEDGMENTS}

This work was supported by the Russian Foundation for Basic Researches under Grant No. 15-02-06031.

[1] T. Idehara, H. Tsuchiya, O. Watanabe, L. Agusu, and S. Mitsudo, The first experiment of a $\mathrm{THz}$ gyrotron with a pulse magnet, Int. J. Infrared Millim. Waves 27, 319 (2007).

[2] M. Yu. Glyavin, A. G. Luchinin, and G. Yu. Golubiatnikov, Generation of 1.5-kW, 1-THz Coherent Radiation from a Gyrotron with a Pulsed Magnetic Field, Phys. Rev. Lett. 100, 015101 (2008).

[3] G. G. Denisov, V. L. Bratman, A. W. Cross, W. He, A. D. R. Phelps, K. Ronald, S. V. Samsonov, and C. G. Whyte, Gyrotron Traveling Wave Amplifier with a 
Helical Interaction Waveguide, Phys. Rev. Lett. 81, 5680 (1998).

[4] J. Pasour, E. Wright, K. T. Nguyen, A. Balkcum, F. N. Wood, R. E. Myers, and B. Levush, Demonstration of a multikilowatt, solenoidally focused sheet beam amplifier at 94 GHz, IEEE Trans. Electron Devices 61, 1630 (2014).

[5] C. D. Joye, A. M. Cook, J. P. Calame, D. K. Abe et al., Demonstration of a high power, wideband $220-\mathrm{GHz}$ traveling wave amplifier fabricated by UV-LIGA, IEEE Trans. Electron Devices 61, 1672 (2014).

[6] G. R. Neil, G. L. Carr, and J. F. Gubeli III, Production of high power femtosecond terahertz radiation, Nucl. Instrum. Methods Phys. Res., Sect. A 507, 537 (2003).

[7] N. Vinokurov, Free electron lasers as a high-power terahertz sources, J. Infrared Millimeter Terahertz Waves 32, 1123 (2011).

[8] G. R. Neil, Accelerator sources for THz science: A review, J. Infrared Millimeter Terahertz Waves 35, 5 (2014).

[9] M. Thomson, M. Kreß, T. Löffler, and H. Roskos, Broadband $\mathrm{THz}$ emission from gas plasmas induced by femtosecond optical pulses: From fundamentals to applications, Laser Photonics Rev. 1, 349 (2007).

[10] I. Babushkin, W. Kuehn, C. Kohler, S. Skupin, L. Berge, K. Reimann, M. Woerner, J. Herrmann, and T. Elsaesser, Ultrafast Spatiotemporal Dynamics of Terahertz Generation by Ionizing Two-Color Femtosecond Pulses in Gases, Phys. Rev. Lett. 105, 053903 (2010).

[11] C. Vicario, B. Monoszlai, and C. P. Hauri, GV/m SingleCycle Terahertz Fields from a Laser-Driven Large-Size Partitioned Organic Crystal, Phys. Rev. Lett. 112, 213901 (2014).

[12] J. Hebling, K.-L. Yeh, M. C. Hoffmann, B. Bartal, and K. A. Nelson, Generation of high-power terahertz pulses by tilted-pulse-front excitation and their application possibilities, J. Opt. Soc. Am. B 25, B6 (2008).

[13] J. A. Fülöp, L. Pálfalvi, M. C. Hoffmann, and J. Hebling, Towards generation of mJ-level ultrashort $\mathrm{THz}$ pulses by optical rectification, Opt. Express 19, 15090 (2011).

[14] H. Hirori, A. Doi, F. Blanchard, and K. Tanaka, Singlecycle terahertz pulses with amplitudes exceeding $1 \mathrm{MV} / \mathrm{cm}$ generated by optical rectification in $\mathrm{LiNbO}_{3}$, Appl. Phys. Lett. 98, 091106 (2011).

[15] N. S. Ginzburg, Pis'ma Zh. Tekh. Fiz. 10, 584 (1984) [Using incoherent pump wave in free electron lasers, Sov. Tech. Phys. Lett. 29, 175 (1984)].

[16] Ya. L. Bogomolov, N.S. Ginzburg, and E. R. Golubyatnikova, Stochastic particle deceleration in the field of an incoherent pump wave or in a random magnetostatic field and effective amplification of monochromatic electromagnetic radiation, Radiophys. Quantum Electron. 38, 183 (1995).

[17] Ya. L. Bogomolov, N.S. Ginzburg, and E. R. Golubyatnikova, Amplification of monochromatic shortwavelength radiation during the stochastic deceleration of a relativistic electron stream in an incoherent pump field, Tech. Phys. 42, 788 (1997).

[18] Ya. L. Bogomolov, N.S. Ginzburg, and E. R. Golubyatnikova, Chaotic particle deceleration and FEL efficiency enhancement with an incoherent pump wave or a stochastic undulator, Nucl. Instrum. Methods Phys. Res., Sect. A 358, 171 (1995).

[19] R. H. Pantell, G. Soncini, and H. E. Puthoff, Stimulated photon-electron scattering, IEEE J. Quantum Electron. 4, 905 (1968).

[20] V. L. Bratman, N. S. Ginzburg, and M. I. Petelin, Energy feasibility of a relativistic Compton laser, JETP Lett. 28, 190 (1978).

[21] V. L. Bratman, N. S. Ginzburg, and M. I. Petelin, Nonlinear theory of stimulated wave scattering by relativistic electron beams, Sov. Phys. JETP 49, 469 (1979).

[22] High Gain, High Power Free Electron Laser: Physics, and Application to TeV Particle Acceleration, edited by $\mathrm{R}$. Bonifacio, L. De Salvo Souza, and C. Pelegrini (North Holland Physics, Amsterdam 1989).

[23] G. Dattoli, A. Renieri, and A. Torre, Lectures on the Free Electron Laser Theory and Related Topics (World Scientific, Singapore, 1993).

[24] I. Pogorelsky, in X-Ray Lasers 2014: Proceedings of the 14th International Conference on X-Ray Lasers, edited by J. Rocca, C. Menoni, and M. Marconi, Springer Proceedings in Physics Vol. 169 (Springer, Berlin, 2016), p. 133.

[25] W. E. Drummond and D. Pines, Non-linear stability of plasma oscillations, Nuclear Fusion, Suppl. 3, 1049 (1962).

[26] A. A. Vedenov, Quasi-linear theory of a plasma, Sov. At. Energy 13, 591 (1963).

[27] A. G. Litvak and V. Yu. Trakhtengerts, Induced scattering of waves and plasma heating by coherent radiation, Sov. Phys. JETP 33, 921 (1971).

[28] T. Taguchi, K. Mima, and T. Mochizuki, Saturation Mechanism and Improvement of Conversion Efficiency of the Free-Electron Laser, Phys. Rev. Lett. 46, 824 (1981).

[29] A. M. Dimos and R. C. Davidson, Quasilinear stabilization of the free electron laser instability for a relativistic electron beam propagating through a transverse helical wiggler magnetic field, Phys. Fluids 28, 677 (1985).

[30] W. P. Marable and P. A. Sprangle, Quasilinear theory of radiation saturation in a free-electron laser, J. Appl. Phys. 67, 3576 (1990).

[31] N. A. Vinokurov, Z. Huang, O. A. Shevchenko, and K. J. Kim, Quasilinear theory of high-gain FEL saturation, Nucl. Instrum. Methods Phys. Res., Sect. A 475, 74 (2001).

[32] A. Renieri, Storage ring operation of the free-electron laser: The amplifier, Il Nuovo Cimento Soc. Ital. Fis. 53B, 160 (1979).

[33] G. Dattoli and A. Renieri, Storage ring operation of the free-electron laser: The oscillator, Il Nuovo Cimento Soc. Ital. Fis. 59B, 1 (1980).

[34] N. S. Ginzburg and M. A. Shapiro, Quasi-linear theory of multimode free-electron lasers with an inhomogeneous frequency broadening, Opt. Commun. 40, 215 (1982).

[35] S. A. Kitsanov, A. I. Klimov, S. D. Korovin, B. M. Kovalchuk, I. K. Kurkan, S. V. Loginov, I. V. Pegel, S. D. Polevin, S. N. Volkov, and A. A. Zherlitsyn, S-band vircator with electron beam premodulation based on compact pulse driver with inductive energy storage, IEEE Trans. Plasma Sci. 30, 1179 (2002). 
[36] N. F. Kovalev, M. I. Petelin, M. D. Raiser, A. V. Smorgonskii, and L.E. Tsopp, Generation of powerful electromagnetic radiation pulses by a beam of relativistic electrons, JETP Lett. 18, 138 (1973).

[37] N. S. Ginzburg, N. I. Zaitsev, E. V. Ilyakov, I. S. Kulagin, Y. V. Novozhilova, R. M. Rozenthal, and A. S. Sergeev, Observation of Chaotic Dynamics in a Powerful Backward-Wave Oscillator, Phys. Rev. Lett. 89, 108304 (2002).

[38] A. V. Arzhannikov, N. S. Ginzburg, G. G. Denisov, P. V. Kalinin, N. Yu. Peskov, A. S. Sergeev, and S. L. Sinitskii, A traveling-wave ring resonator with Bragg deflectors in a two-stage terahertz free-electron laser, Tech. Phys. Lett. 40, 730 (2014).

[39] A. V. Arzhannikov, N. S. Ginzburg, V. Yu. Zaslavsky, P. V. Kalinin, N. Yu. Peskov, A. S. Sergeev, S. L. Sinitsky, V. D. Stepanov, and M. Thumm, Generation of powerful narrow-band $75-\mathrm{GHz}$ radiation in a free-electron maser with two-dimensional distributed feedback, Tech. Phys. Lett. 39, 801 (2013).

[40] A. V. Arzhannikov, N. S. Ginzburg, P. V. Kalinin, A. M. Malkin, N. Yu. Peskov, A. S. Sergeev, S. L. Sinitsky, M. Thumm, and V. Yu. Zaslavsky, Short-wavelength tunable Bragg reflectors based on coupling of propagating and cutoff waves: Modeling and experimental studies, Appl. Phys. Lett. 101, 083507 (2012).

[41] N. S. Ginzburg, A. M. Malkin, N. Yu. Peskov, A. S. Sergeev, V. Yu. Zaslavsky, and I. V. Zotova, Powerful terahertz free electron lasers with hybrid Bragg reflectors, Phys. Rev. ST Accel. Beams 14, 042001 (2011).

[42] I. V. Yakovlev, Stretchers and compressors for ultrahigh power laser systems, Quantum Electron. 44, 393 (2014). 